\title{
The Origin of the Giant Hall Effect in Metal-Insulator Composites*
}

\author{
Joachim Sonntag\# \\ MEAS Deutschland GmbH a TE Connectivity LTD Company, Dortmund, Germany \\ Email: "sonntag.joachim45@gmail.com
}

Received 7 April 2016; accepted 28 June 2016; published 1 July 2016

Copyright @ 2016 by author and Scientific Research Publishing Inc.

This work is licensed under the Creative Commons Attribution International License (CC BY). http://creativecommons.org/licenses/by/4.0/

cc) (i) Open Access

\section{Abstract}

Near the metal-insulator transition, the Hall coefficient $R$ of metal-insulator composites (M-I composite) can be up to $10^{4}$ times larger than that in the pure metal called Giant Hall effect. Applying the physical model for alloys with phase separation developed in [1] [2], we conclude that the Giant Hall effect is caused by an electron transfer away from the metallic phase to the insulating phase occupying surface states. These surface states are the reason for the granular structure typical for M-I composites. This electron transfer can be described by $-d n=\beta \cdot n \cdot d\left(\frac{v_{B}}{v_{A}}\right)$ [1] [2], provided that long-range diffusion does not happen during film production ( $n$ is the electron density in the phase $A . v_{A}$ and $v_{B}$ are the volume fractions of the phase $A$ (metallic phase) and phase $B$ (insulator phase). $\beta$ is a measure for the average potential difference between the phases $A$ and $B$ ). A formula for calculation of $R$ of composites is derived and applied to experimental data of granular $\mathrm{Cu}_{1-y}\left(\mathrm{SiO}_{2}\right)_{y}$ and $\mathrm{Ni}_{1-y}\left(\mathrm{SiO}_{2}\right)_{y}$ films.

\section{Keywords}

Metal-Insulator Composites, Granular Metals, Hall Coefficient, Conductivity, Electron Density

\section{Introduction}

Nanocomposites play a growing role in both scientific research and practical applications because of the possibility of combination of special properties which cannot be reached in classical materials [3]-[5]. A prominent example for both scientific challenge and practical application is the Giant Hall effect (GHE) in metal-insulator

*PACS numbers: 71.23.-k 71.55.Jv 72.10.Bg 72.15.-v.

${ }^{\#}$ Corresponding author. 
composites (M-I composites): Near the metal-insulator transition (M-I transition), the Hall coefficient can be up to $10^{4}$ times larger than that in the pure metal [6]-[16].

Applications of the GHE we find in magnetic field sensing elements, in read heads of magnetic recording devices and magnetic switching devices. Other examples for practical applications of nanocomposites are biomedical ones, materials with improved corrosion resistance, and thermoelectric materials with higher efficiency for energy harvesting, environmentally friendly refrigeration, direct energy transformation from heat into electricity, and temperature sensors.

As reasons for the GHE, quantum size effects and quantum interference effects on the mesoscopic scale have been discussed [8] [11]-[14] [17]. To our knowledge, until now, there is no explanatory model which can interpret the phenomenon of GHE. In the present paper, we present a discussion of the reasons for the GHE applying the electron transfer model [1] [2] developed for metal-metalloid alloys. This model can be summarized by three points ${ }^{1}$ :

For large ranges of concentration there is

(1) Phase separation between two phases called phase $A$ and phase $B$, where each phase has its "own" short-range order (SRO),

(2) The phase separation leads to band separation in the conduction band (CB) and valence band (VB) connected with the phases $A$ and $B$, respectively, and the electrons are freely propagating and the corresponding wave functions are extended over connected regions of one phase as long as the phase forms an infinite (macroscopic) cluster through the alloy.

(3) Between the two coexisting phases there is electron redistribution (electron transfer) which can be described by

$$
n(\zeta)=n_{A} \cdot \exp (-\beta \zeta)
$$

where $\zeta$ is the quotient of the volume or atomic fractions ${ }^{2}$ of the two coexisting phases. $n(\zeta)$ is the electron density in the phase $A$ with $n_{A}=n(0) . \quad \beta$ is a constant for a given alloy, which is determined by the average potential difference between the two phases.

The points (1) and (2) imply the fact that each phase can be characterized by its own transport coefficients which can be calculated, in principle, by classical transport theory as done in [2] (conductivity) and [18] [19] (Seebeck coefficient).

Since M-I composites also consist of two separate phases with phase grains at the nanoscale, it is obvious to ask whether Equation (1) is reflected in the concentration dependence of the Hall coefficient $R$ of M-I composites as well. Indeed, we have found that in the metallic regime of $\mathrm{Cu}_{1-y}\left(\mathrm{SiO}_{2}\right)_{y}$ and $\mathrm{Ni}_{1-y}\left(\mathrm{SiO}_{2}\right)_{y}$ thin films, the concentration dependence of $R$ can be approximated by linear relations

$$
d \ln |R|=\alpha^{\prime} \cdot d \eta
$$

with constant slope $\alpha^{\prime}$. For $\mathrm{Cu}_{1-y}\left(\mathrm{SiO}_{2}\right)_{y}$ and $\mathrm{Ni}_{1-y}\left(\mathrm{SiO}_{2}\right)_{y}$ it follows from Figure 1(a) and Figure 1(b), $\alpha^{\prime}=7.9$ and $\alpha^{\prime}=10.3$ with the coefficient of determination $r^{2}=0.92$ and $r^{2}=0.96$, respectively. $\eta=y /(1-y)$, where $y$ is the volume fraction of $\mathrm{SiO}_{2}$. This finding is illustrated in Figure 1(a) and Figure 1(b), where the absolute $R$ values measured by Zhang et al. [12], Saviddes et al. [20] and Pakhomov et al. [10] are drawn versus $\eta$. The signs of the $R$ values are negative. $\mathrm{For} \mathrm{Ni}_{1-y}\left(\mathrm{SiO}_{2}\right)_{y}$, Figure 1 (b), the extraordinary $R$ values (taken from Fig. 3 in [10]) are drawn.

Figure 1(a) and Figure 1(b) reflect immediately Equation (1) provided that $|R| \propto 1 / n$ (nearly free electrons $\mathrm{NFE}$ ). For a more precise discussion, we have to separate the contribution of the metallic phase to $R$, which can be done applying effective medium theory (EMT, [2], Sec. IVA therein).

The known EMT-formula for the Hall coefficient derived by Cohen and Jortner [21] is

$$
\sum_{i=A, B} v_{i} \cdot \frac{\sigma_{i}^{2} R_{i}-\sigma^{2} R}{\left(\sigma_{i}+2 \sigma\right)^{2}}=0
$$

\footnotetext{
${ }^{1}$ The points (1) and (2) are now confirmed experimentally or supported by independent authors [39]-[50] (details in [2], Sec. I therein). Support for point (3) comes from the fact that it is successfully applied for a quantitative description of the concentration dependence of both conductivity and Seebeck coefficient in [18] [19] and of the M-I transition and structural features of metal-metalloid alloys and M-I composites in [2].

${ }^{2}$ In [1], the available experimental data were not sufficient to decide this question. This question was discussed in [2] with the result that $\zeta$ is to be interpreted as volume quotient.
} 


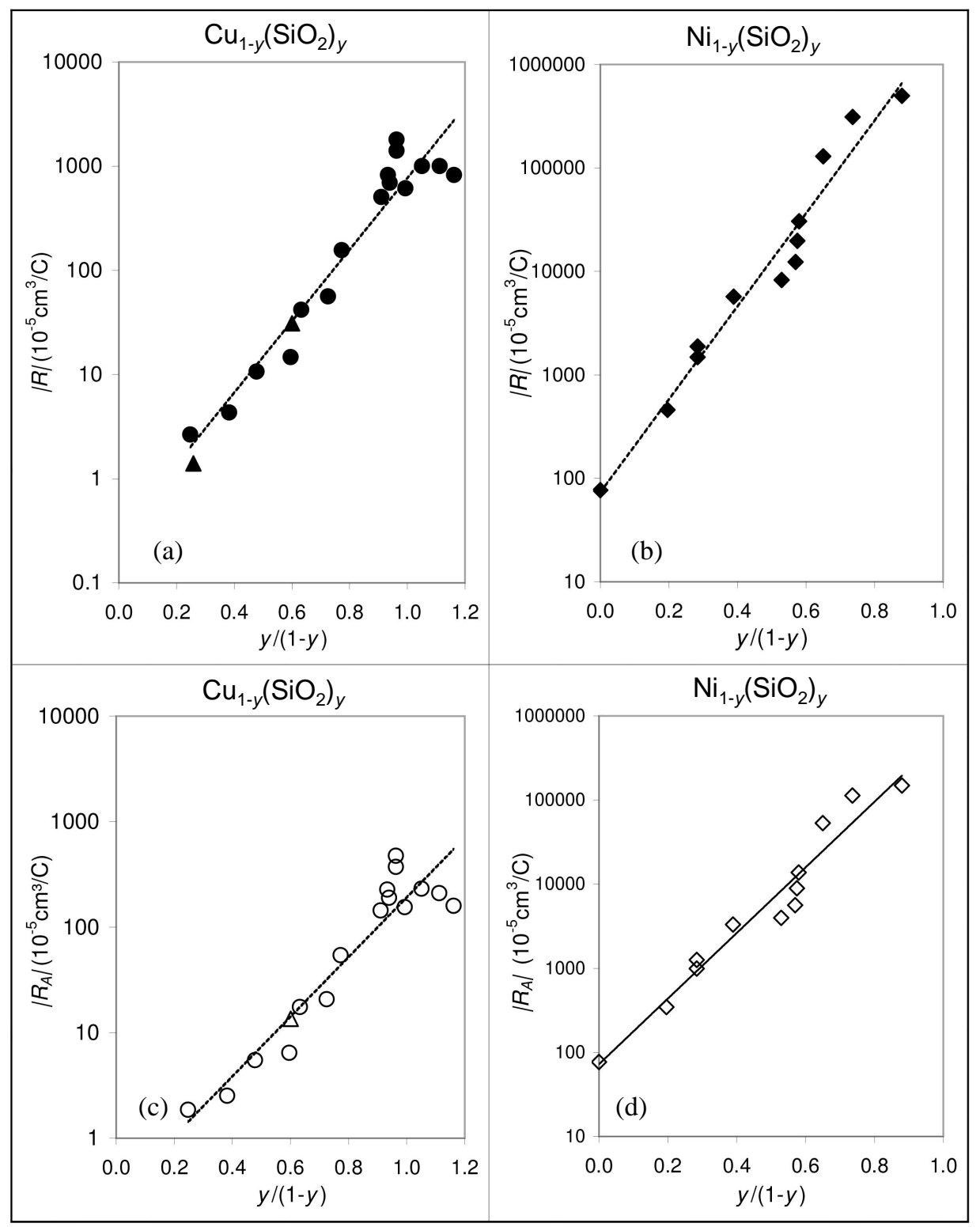

Figure 1. Experimental Hall coefficient data at $5 \mathrm{~K}$ versus $y /(1-y)$ for $\mathrm{Cu}_{1-y}\left(\mathrm{SiO}_{2}\right)_{y}$, (a), (c), and $\mathrm{Ni}_{1-y}\left(\mathrm{SiO}_{2}\right)_{y}$, (b), (d), taken from [12] (circles), [20] (triangles) and [10] (diamonds). (c), (d): $R_{A}$ calculated by $R_{A}=R \frac{\left(3 v_{A}-1\right)}{2}$ according to Equation (16), where $v_{A}=1-y$ is set.

where $\sigma$ and $R$ are the electrical conductivity and Hall coefficient of a composite, respectively. $\sigma_{i}$ and $R_{i}$ are the corresponding transport parameters of the phase $i .^{3} v_{i}$ is the volume fraction of the phase $i(i$ stands for the phase $A$ or $B$ ).

As will be argued in Sec. 3.1, Equation (3) seems to be a good approximation for two-phase composites if $\sigma_{A} \approx \sigma_{B}$, but not if $\sigma_{A} \gg \sigma_{B}$, as typical for M-I composites. Therefore, in Sec. 2 a $R$ formula will be derived which holds for $\sigma_{A} \gg \sigma_{B}$ as well. In Sec. 3.1 this $R$ formula and Equation (3) will be compared and its applicability to M-I composites will be checked. In Sec. 3.2 it will be applied to a quantitative discussion of the GHE in M-I composites. In Sec. 3.3 the effect of the grain size on the GHE will be discussed. In Sec. 4 the results will be summarized.

\footnotetext{
${ }^{3}$ Equation (3) is a comprehensive formulation of the Equations (16)-(20) of [21].
} 


\section{Derivation of the $R$ Formula}

Let us consider a non-magnetic two-phase composite, where the phase grains are spherical without preferred orientations and arranged in a symmetrical fashion and each phase $i$ can be characterized by a set of transport coefficients. The local electric current density in a single grain of the phase $i(i=A$ or $B)$ can be written as

$$
\boldsymbol{J}_{i}=\stackrel{\leftrightarrow}{\sigma_{i}} \boldsymbol{E}_{i},
$$

where $\boldsymbol{E}_{i}$ and ${\stackrel{\leftrightarrow}{\sigma_{i}}}_{i}$ are the electric field and the magnetoconductivity tensor [22] in this grain. For the electric current density outside of this grain we write analogously

$$
\boldsymbol{J}=\stackrel{\leftrightarrow}{\sigma} \boldsymbol{E}
$$

where $\boldsymbol{E}$ and $\stackrel{\leftrightarrow}{\sigma}$ are the electric field and the magnetoconductivity tensor outside of this grain (effective medium). For the determination of the coefficients in $\sigma_{i}$ we start with the equation for $\boldsymbol{J}_{i}$ under the influence of an electrical and magnetic field, [23]-[25]

$$
\boldsymbol{J}_{i}=e_{i}^{2} K_{11, i} \boldsymbol{E}_{i}+\frac{e_{i}^{3}}{m_{i}} K_{12, i}\left(\boldsymbol{E}_{i} \times \boldsymbol{B}\right)+\frac{e_{i}^{4}}{m_{i}^{2}} K_{13, i} \boldsymbol{B}\left(\boldsymbol{E}_{i} \cdot \boldsymbol{B}\right) .
$$

$K_{r s, i}$ are the transport integrals, $e_{i}=-|e|$ and $+|e|$ for electrons and holes, respectively. $|e|$ is the elementary charge. The third summand in Equation (6) disappears only if $\boldsymbol{E}$ (or $\boldsymbol{E}_{i}$ ) is always perpendicular to $\boldsymbol{B}$. In a composite, however, $\boldsymbol{B}$ and $\boldsymbol{E}$ (or $\boldsymbol{E}_{i}$ ), are generally not perpendicular to each other because of the spherical boundary between a phase grain and its surroundings. Without loss of generality, the external fields applied to the sample, $\boldsymbol{E}_{\text {ext }}$ and $\boldsymbol{B}$, have the directions of the $X$ and $Z$ axes, respectively. Then Equation (6) and Equation (4) lead to

$$
\stackrel{\leftrightarrow}{\sigma_{i}}=\frac{\sigma_{i}}{1+\xi_{i}^{2}}\left(\begin{array}{ccc}
1 & \xi_{i} & 0 \\
-\xi_{i} & 1 & 0 \\
0 & 0 & 1+v_{i} \xi_{i}^{2}
\end{array}\right),
$$

where $\xi_{i} \equiv \mu_{i} B=\sigma_{i} R_{i} B$. Analogously we write for $\stackrel{\leftrightarrow}{\sigma}$,

$$
\stackrel{\leftrightarrow}{\sigma}=\frac{\sigma}{1+\xi^{2}}\left(\begin{array}{ccc}
1 & \xi & 0 \\
-\xi & 1 & 0 \\
0 & 0 & 1+v \xi^{2}
\end{array}\right)
$$

with $\xi \equiv \mu B=\sigma R B . \quad v_{i}=1+\cos \gamma_{i}$ and $v=1+\cos \gamma$, where $\gamma_{i}$ and $\gamma$ are the angle between $\boldsymbol{E}_{i}$ and $\boldsymbol{B}$, respectively between $\boldsymbol{E}$ and $\boldsymbol{B} . \mu$ and $\mu_{i}$ are the Hall mobility in the composite and phase $i$, respectively.

At the interface between a single phase grain and its surroundings continuity of the normal components of the current density and the tangential components of the potential gradient are to be fulfilled. For the limiting case $\boldsymbol{B}=0$, this demand is fulfilled by

$$
f\left(\sigma, \sigma_{i}\right) \equiv \sigma_{A} \sigma_{B}+\sigma \sigma_{A}\left(3 v_{A}-1\right)+\sigma \sigma_{B}\left(3 v_{B}-1\right)-2 \sigma^{2}=0
$$

following from the EMT-formula for $\sigma$, [27] [28]

$$
\sum_{i} v_{i} \frac{\sigma_{i}-\sigma}{\sigma_{i}+2 \sigma}=0
$$

For the case $\boldsymbol{B} \neq 0$, the tensor properties of $\stackrel{\leftrightarrow}{\sigma_{i}}$ and $\stackrel{\leftrightarrow}{\sigma}$, Equation (7) and Equation (8), are to be taken into account. Equation (9) expressed in tensor form reads

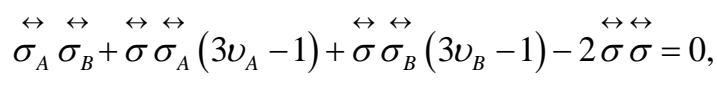

where the identities $\stackrel{\leftrightarrow}{\sigma_{A}} \stackrel{\leftrightarrow}{\sigma_{B}}=\stackrel{\leftrightarrow}{\sigma_{B}} \stackrel{\leftrightarrow}{\sigma_{A}}$ and $\stackrel{\leftrightarrow}{\sigma} \stackrel{\leftrightarrow}{\sigma}_{i}=\stackrel{\leftrightarrow}{\sigma_{i}} \stackrel{\leftrightarrow}{\sigma}$ have been used. Equation (11) determines the coeffi- 
cients of Equation (8) as a function of the coefficients of Equation (7). Inserting Equation (7) and Equation (8) into Equation (11) and comparing coefficients for the tensor elements, we get

$$
\xi=\frac{\sigma_{A} \sigma_{B}\left(\xi_{A}+\xi_{B}\right)+\sigma \sigma_{A} \xi_{A}\left(3 v_{A}-1\right)+\sigma \sigma_{B} \xi_{B}\left(3 v_{B}-1\right)}{4 \sigma^{2}-\sigma \sigma_{A}\left(3 v_{A}-1\right)-\sigma \sigma_{B}\left(3 v_{B}-1\right)},
$$

following from the tensor elements $\sigma_{x y}$ or $\sigma_{y x}$, where quadratic and higher powers of $\xi, \xi_{i}$ are neglected, i.e., Equation (12) and the following Equations (13), (14) are low-field approximations. Within this approximation the parameters $v_{i}$ and $v$ do not have an influence on the result. From the tensor elements $\sigma_{x x}$, $\sigma_{y y}$, or $\sigma_{z z}$, Equation (10) follows.

Substituting $\xi$ and $\xi_{i}$ in Equation (12) by $R$ and $R_{i}$ and considering Equation (9) we get the $R$ formula for two-phase composites:

$$
R=\frac{\sigma_{A}^{2} R_{A}\left[\sigma_{B}+\sigma\left(3 v_{A}-1\right)\right]+\sigma_{B}^{2} R_{B}\left[\sigma_{A}+\sigma\left(3 v_{B}-1\right)\right]}{\sigma\left(\sigma_{A} \sigma_{B}+2 \sigma^{2}\right)} .
$$

The same formalism can also be applied to composites with more than two phases leading to relatively complex formulae for $R$. A self-contained and more manageable description of these $R$ formulae is given by

$$
\left(R \sigma^{2} \frac{\partial}{\partial \sigma}+\sum_{i=A, B, \cdots} R_{i} \sigma_{i}^{2} \frac{\partial}{\partial \sigma_{i}}\right) f\left(\sigma, \sigma_{i}\right)=0
$$

with

$$
f\left(\sigma, \sigma_{i}\right)=\left(\prod_{i=A, B, \cdots}\left(\sigma_{i}+2 \sigma\right)\right)\left(\sum_{i=A, B, \cdots} v_{i} \frac{\sigma_{i}-\sigma}{\sigma_{i}+2 \sigma}\right) .
$$

\section{Discussion}

\subsection{Comparison between Equation (3) and Equation (13)}

For three examples of two-phase composites, in Figure 2(b), Figure 2(d), and Figure 2(f), the concentration dependence of $R$ related to its values at $v_{A}=1$ is shown, calculated by Equation (13), and compared with Equation (3), denoted as " $C$ \& J". In Figure 2(a), Figure 2(c), and Figure 2(e), the corresponding concentration dependence of the Hall mobility $\mu(=\sigma R)$ is shown, where $\sigma$ is calculated by Equation (9). There are two essential differences between the two solutions Equation (3) and Equation (13):

(1) The most striking difference appears in Figure 2(a) and Figure 2(c): The "C \& J" curves decrease dramatically with increasing $v_{A}$ and pass through a pronounced minimum at $v_{A}=1 / 3$, although $\mu_{A}=\mu_{B}$ and $\mu_{A}>\mu_{B}$, respectively. In contrast, the $\mu$ curves calculated by Equation (13) agree with the expectation: Figure 2(a): $\mu$ agrees with $\mu_{A}$ for all $v_{A}$; Figure 2(c) and Figure 2(e): $\mu$ increases and decreases with increasing $v_{A}$, respectively.

A possible interpretation for such dramatic decrease of $\mu$ at $v_{A}=1 / 3 \quad$ (" $\mathrm{C} \& \mathrm{~J}$ " curves) could be additional scattering centres in the added phase boundaries. Such an effect by the phase boundaries is expected to be the more pronounced the smaller the sizes of the phase grains, $D_{i}$. However, the C \& J formula [21] [26] does not contain $D_{i}$.

The differences between Equation (13) and the curves " $C \& J$ " are the larger the larger the difference between $\sigma_{A}$ and $\sigma_{B}$. On the other hand, for the limiting case, $\sigma_{A}=\sigma_{B}$, Equation (3) and Equation (13) agree.

(2) Another striking difference between Equation (13) and Equation (3) is represented by the boundary case “ $\sigma_{B}=0$ and $\sigma_{A} \neq 0$ ”, for which one obtains

$$
\frac{1}{R^{(13)}}=\frac{1}{R_{A}} \frac{\left(3 v_{A}-1\right)}{2},
$$

and

$$
\frac{1}{R^{\mathrm{C} \& \mathrm{~J}}}=\frac{1}{R_{A}} \frac{\left(3 v_{A}+1\right)}{4},
$$




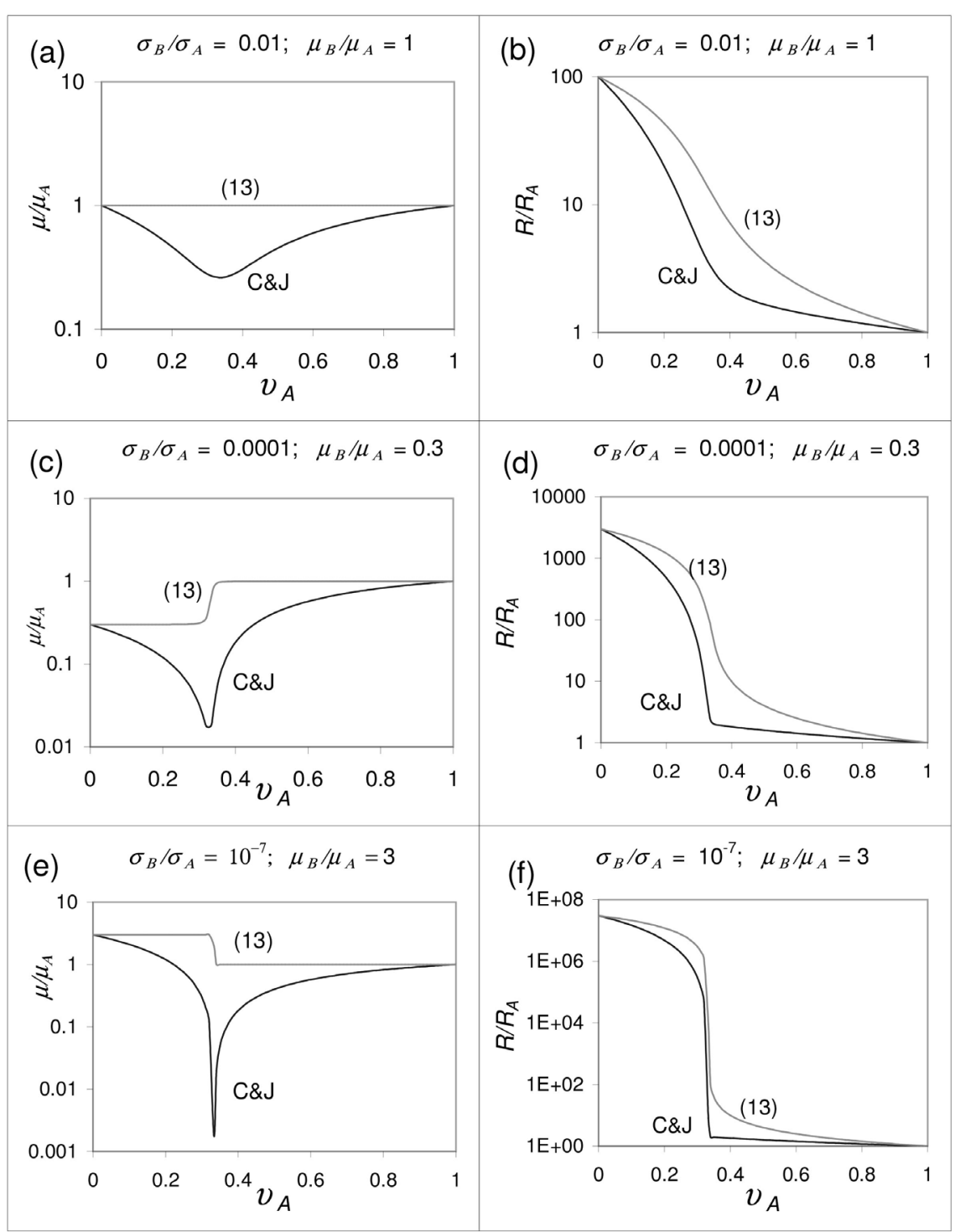

Figure 2. $\mu / \mu_{A}$ and $R / R_{A}$ versus $v_{A}$ calculated by Equation (3) ("C \& J") and by Equation (13). The "C \& J"-curves in Figure 2(a) and Figure 2(c) agree with the curves "5" and "7" shown in Fig. 1(b), Fig. 1 (c) of [21] and Fig. 13, Fig. 14 of [26], where the same examples are chosen.

respectively, and for $\sigma$, Equation (9) gives

$$
\sigma=\sigma_{A} \frac{\left(3 v_{A}-1\right)}{2}
$$

Starting at $v_{A}=1$, with decreasing $v_{A}$ both $\sigma$ and $\frac{1}{R^{(13)}}$ decrease continuously until they vanish at $v_{A}=1 / 3$. This result corresponds to the fact that for $v_{A}<1 / 3$ there is no longer a connected metal cluster through the composite (in correspondence with the assumption made earlier that the phase grains are spherical without preferred orientations and arranged in a symmetrical fashion). This result is, however, not reflected by Equation (17) which gives $\frac{1}{R^{\mathrm{C \& J}}}>0$ even for $v_{A}<1 / 3$, where all the metallic granules are separated by adjacent insulating phase regions, that is, electron transport through the sample does not happen, if additional 
tunneling is excluded.

These two differences, (1) and (2), suggest the fact that Equation (16) represents the physical situation better than Equation (17). Therefore, in the following, Equation (13), respectively Equation (16), will be applied in a discussion of the Hall coefficient in M-I composites.

\subsection{The Giant Hall Effect in M-I Composites}

For $R_{A}$ calculated by Equation (16) applied to the $R$ data of Figure 1(a), Figure 1(b), we find that they can be approximated by a relation similar to Equation (2),

$$
d \ln \left|R_{A}\right|=\beta^{\prime} \cdot d \eta,
$$

where $\beta^{\prime}$ is a constant for a given M-I composite: For $\mathrm{Cu}_{1-y}\left(\mathrm{SiO}_{2}\right)_{y}$ and $\mathrm{Ni}_{1-y}\left(\mathrm{SiO}_{2}\right)_{y}$ it follows from Figure 1 (c) and Figure 1(d), $\beta^{\prime}=6.5$ and $\beta^{\prime}=9.0$ with the coefficient of determination $r^{2}=0.89$ and $r^{2}=0.95$, respectively. ${ }^{4}$ This finding suggests that the colossal increase of $|R|$ is caused by one (!) effect acting in the complete metallic regime. Inserting $\left|R_{A}\right| \propto 1 / n$ (NFE approximation) in Equation (19) leads to Equation (1), or in differential form,

$$
-d n=\beta \cdot n \cdot d \zeta,
$$

where $\beta \approx \beta^{\prime} . n$ is the electron density in the metallic phase and $\zeta=v_{B} / v_{A} . v_{B}$ and $v_{A}$ are the volume fractions of the insulator phase $(B)$ and metallic phase $(A)$, respectively. $v_{B}$ and $v_{A}$ are identical with $y$ and $1-y$, respectively, if the insulating phase consists only of $\mathrm{SiO}_{2}$ and the metallic phase only of $\mathrm{Cu}$ or $\mathrm{Ni}$. In this case, $\beta=\beta^{\prime}$. If, however, a certain portion of the metalloid atoms is dissolved in the metallic phase and/or a certain portion of the metal atoms is solved in the insulating phase, then $\beta^{\prime}$ is only an approximation for $\beta$. Equations (1) and (20) agree with the equations (15a) and (15b) in [1], respectively, which describe electron transfer between the phases in amorphous transition-metal-metalloid alloys. ${ }^{5}$ There the parameter $\beta$ was interpreted to be a constant for a given composite, which is determined by the average potential difference between the phases, $\Delta V .^{6}$ Phase $B$ is the phase with the deeper potential. Because of this analogy, Equation (19) suggests the following interpretation of the GHE: The colossal increase of $|R|$ with decreasing metal content is essentially caused by a decrease of $n$ due to electron transfer to the insulator phase $\left(\mathrm{SiO}_{2}\right)$ which can be described by Equation (1), respectively Equation (20).

Because the Fermi level lies in the energy gap between the valence band and conduction band of the insulator $\mathrm{SiO}_{2}$ phase, the transferred electrons occupy surface states on the $\mathrm{SiO}_{2}$ phase. This is the reason for the granular structure: spherical metal grains are embedded in the amorphous $\mathrm{SiO}_{2}$ phase (see, e.g., [29], Figs. 13-16 therein). A minimum energy is realized if, firstly, the transferred (pinned) electrons are arranged on spherical surfaces and, secondly, the insulating phase forms very thin layers around the metal grains providing the largest possible surface to accommodate the large number of transferred electrons. This electron transfer from the metallic phase to the phase boundaries provides the logical explanation for the granular structure in M-I composites. Such a granular structure has been found in many M-I films [7] [13] [15] [29]. This proposal applies to magnetic M-I composites as well. For nonmagnetic M-I composites the parameter $C$ in

$$
R_{A}=-\frac{C}{|e| \cdot n}=\frac{\mu_{A}}{\sigma_{A}}
$$

(NFE approximation) is of the order of one, depending slightly on the magnetic field. [23] [24] $\sigma_{A}$ and $\mu_{A}$ are the conductivity and Hall mobility, respectively, of the phase $A .|e|$ is the elementary charge. For magnetic M-I composites Equation (21) holds approximately if " $=$ " is replaced by " $\propto$ ” considering the effect of the additional internal magnetic field due to the magnetization: An electron sees the effective magnet field $H_{w}=H+H_{i}$, where $H_{i} \gg H . H$ is the external field applied to the specimen and $H_{i}$ is the internal field produced by the quantum mechanical exchange forces ([30], p. 341). An electron does not distinguish between $H$ and $H_{i}$. It moves according to the Lorentz force determined by $H_{w}$ and the electrical field $E$. One can as${ }^{4} R_{A}$ calculated by Equation (17) can also be approximated by Equation (19) with the slopes $\beta^{\prime}=7.5$ and $\beta^{\prime}=9.9$ and $r^{2}=0.92$ and $r^{2}=0.96$, for $\mathrm{Cu}_{1-\mathrm{y}}\left(\mathrm{SiO}_{2}\right)_{\mathrm{y}}$ and $\mathrm{Ni}_{1-\mathrm{y}}\left(\mathrm{SiO}_{2}\right)_{\mathrm{y}}$, respectively.

${ }^{5}$ For large ranges of composition, amorphous transition-metal—metalloid alloys are composed of different amorphous phases [39]-[43] with $D_{i} \sim 1$ - 2 nm [42] [43].

${ }^{6}$ The potential difference $\Delta V$ is identical with the difference of the electrochemical potentials of the phases, as long as they are not in contact to each other. Only, when a contact is realized, a common electrochemical potential is realized by electron transfer between the phases. 
sume that $H_{w}$ is nearly proportional to $H$ as long as $H_{i}$ is nearly proportional to the magnetization produced by $H$.

This assumption is supported by the experimental finding by Xiong et al. [31] that (for not too small fields $H$ ), in the granular Co-Ag system, the Hall resistivity $\rho_{x y}$ is linearly proportional to $H$. If so, the measured $R$ values differ from the calculated $R$ values, Equation (21), only by a factor which is nearly constant. Therefore, we assume that the EMT-formula for $R$, Equation (13), can be applied to magnetic composites as well.

If the metallic phase of a M-I composite is a noble metal, the NFE approximation is a good one for the metallic phase, above all as the Fermi surface moves away from the Brillouin zone boundary as $n$ decreases. For the metallic phase in $\mathrm{Ni}_{-} \mathrm{SiO}_{2}$ the NFE approximation is surely also a good one, because Ni has only 0.55 ss valence electrons per $\mathrm{Ni}$ atom ([30], p. 271).

If the metallic phase of a M-I composite is a transition-metal, the electron transfer is expected to be composed of both the $d$ and $s$ electrons. As the $d$ density of states at the Fermi level is essentially larger than the s density of states, the principal share of electrons transferred to the insulating phase, is made up of $d$ electrons, that is, the $s$ electron density in the metallic phase remains relatively large. Because the electronic transport is determined by the $s$ valence electrons in the $A$ phase, the effect of the electron transfer on the electronic transport in the metallic phase is expected to be relatively small, and the increase of $R_{A}$ due to electron transfer should be essentially smaller as in M-I composites containing a noble metal as metallic phase. For instance, in $\mathrm{Mo}_{1-y}\left(\mathrm{SnO}_{2}\right)_{y}([7]$, Fig. 2 therein), we do not find an exponential change of $R_{A}$ with increasing $y /(1-y)$ : for $0<y<0.55$ (i.e. $0<y /(1-y)<1.22$ ), the experimental $R$ values [7] of $\mathrm{Mo}-\mathrm{SnO}_{2}$ fluctuate slightly where the average of $R_{A}$ calculated by Equation (16) remains nearly independent of $y$. Only approaching the M-I transition $(y>0.6), R_{A}$ increases drastically.

Now the question arizes: why do we find an exponential dependence of $n(\zeta)$ in $\mathrm{Ni}_{1-y}\left(\mathrm{SiO}_{2}\right)_{y}$ although Ni is a transition-metal? X-ray emission spectra of amorphous and crystalline $\mathrm{Ni}_{1-y} \mathrm{Si}_{y}$ and $\mathrm{Pd}_{1-y} \mathrm{Si}_{y}$ alloys by Tanaka et al. [32] have shown that there are strong bonds between $d$ orbitals (of $\mathrm{Ni}$ and $\mathrm{Pd}$ ) and $\mathrm{Si} p$ orbitals leading to a stronger splitting of the $d$ band into a bonding and antibonding fraction, where the former is lifted, whereas the latter lies below the Fermi level. Analogously, for $\mathrm{Ni}_{1-y}\left(\mathrm{SiO}_{2}\right)_{y}$ one can also expect strong bonds between Ni $d$ orbitals and Si (and O) $p$ orbitals which leads to a strong reduction or disappearance of the $d$ density of states at the Fermi level. Therefore, we find an experimental increase of $\left|R_{A}\right|$ (Figure 1(d)). Moreover, there is strong evidence for the assumption that the metallic phase does not consist of $\mathrm{Ni}$ alone, but that there is a certain fraction of Si (and $\mathrm{O}$ atoms) dissolved in the metallic phase.

In summary, for M-I composites containing a noble metal, we expect an exponential $n(\zeta)$ dependence because the electron transfer is made up entirely of the $s$ electron density. For M-I composites containing a transition-metal, an exponential $n(\zeta)$ dependence can be expected if the $d$ density of states at the Fermi level is strongly reduced, for instance caused by a hybridization of the $d$ states with the $p$ states of the metalloid.

Comparing granular M-I composites with amorphous transition-metal-metalloid alloys ([1]), we state that the exponential increase of $R$ and the exponential decrease of $\sigma$ with $y$ (respectively $y /(1-y)$ ) is essentially caused by the same phenomenon: decrease of the electron density in the metallic phase due to electron transfer to the metalloid or insulator phase. The essential difference between these two material classes is the fact that in the metalloid phase of the amorphous transition-metal-metalloid alloys an incompletely occupied $s p$ band can exist ([2], Sec. IIA therein) for accepting the transferred electrons. In contrast, in the insulator phase of M-I composites only localized states on the surface of it are available for acceptance of the transferred electrons. This difference is also the reason for the different microscopic structures of M-I composites and amorphous transition-metal-metalloid alloys. Another, rather quantitative difference is the fact that the decrease of $n$ is essentially larger than in amorphous transition-metal-metalloid alloys, as the average potential difference between the phases, $\Delta V$, is essentially larger.

Our electron transfer model is compatible with a series of other experimental findings:

1) The GHE occurs both in magnetic M-I composites and non-magnetic ones suggesting a mechanism independent from magnetism [13].

2) In M-I composites, $\sigma$ and $\sigma_{A}$ decrease exponentially with decreasing metal content in correspondence with the exponential increase of $R$. For some M-I composites, in Figure 3, $\log [\sigma(y) / \sigma(0)]$ and $\log \left[\sigma_{A}(y) / \sigma_{A}(0)\right]$ are drawn versus $y /(1-y)$. In the NFE approximation the connection between $\sigma_{A}$ and $n$ is given by

\footnotetext{
${ }^{7} \mathrm{In} \mathrm{Mo}_{l-y}\left(\mathrm{SnO}_{2}\right)_{y}$ the carriers are holes [7]; electron transfer away from the metallic phase can lead to an increase of the hole density $p$, but also to a decrease of it depending on the position of the Fermi surface in relation to the Brillouin zones.
} 

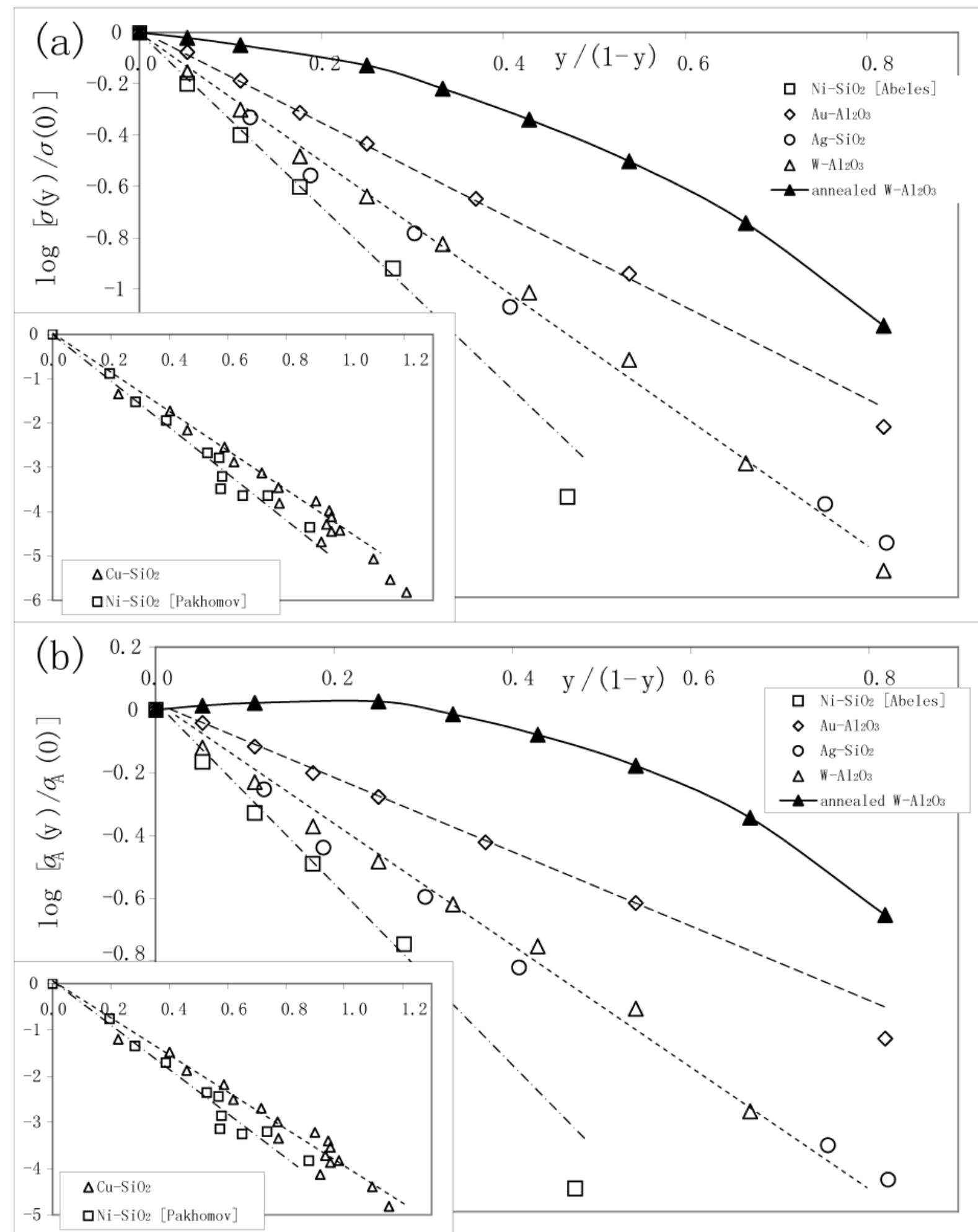

Figure 3. (a) $\log [\sigma(y) / \sigma(0)]$ versus $y /(1-y)$ for $\mathrm{Ni}_{1-y}\left(\mathrm{SiO}_{2}\right)_{y}, \mathrm{Au}_{1-y}\left(\mathrm{Al}_{2} \mathrm{O}_{3}\right)_{y}, \mathrm{~W}_{1-y}\left(\mathrm{Al}_{2} \mathrm{O}_{3}\right)_{y}$ and annealed $\mathrm{W}_{1-y}\left(\mathrm{Al}_{2} \mathrm{O}_{3}\right)_{y}$ (at $1200^{\circ} \mathrm{C}$ in $\mathrm{H}_{2}$ ) taken from Abeles et al. ([29], Fig. 19 therein) and $\operatorname{Ag}_{1-y}\left(\mathrm{SiO}_{2}\right)_{y}$, Priestley et al. [33]. (b) $\log \left[\sigma_{A}(y) / \sigma_{A}(0)\right]$ versus $y /(1-y)$, calculated by Equation (18) and $v_{A}=1-y$. Inlets in (a) and (b): $\mathrm{Cu}_{1-y}\left(\mathrm{SiO}_{2}\right)_{y}$ and $\mathrm{Ni}_{1-y}\left(\mathrm{SiO}_{2}\right)_{y}$, taken from Liu et al. [14] and Pakhomov et al. [10], respectively. 


$$
\sigma_{A}=2\left(\frac{\pi}{3}\right)^{1 / 3} \frac{e^{2}}{h} \operatorname{Ln}^{2 / 3}=|e| \mu_{A} n,
$$

where $\mu_{\mathrm{A}}$ is the mobiliy of the carriers which is assumed to be equal to the Hall mobility introduced in Sec. 2 . $h$ is Plancks constant. $L$ is the (elastic) mean free path of the electronic carriers in the (metallic) phase A. Because of Equation (22) the exponential concentration dependence of $n$, Equation (1), is also reflected by the concentration dependence of $\sigma_{A}(y) / \sigma_{A}(0)$ in Figure 3 if the concentration dependences of $L$ or $\mu_{A}$ can either be neglected or change exponentially with $y /(1-y)$ as well. For $\mathrm{W}_{1-y}\left(\mathrm{Al}_{2} \mathrm{O}_{3}\right)_{y}$ we assume that there are strong bonds between $\mathrm{W} d$ orbitals and $\mathrm{Si}$ (and O) $p$ orbitals, comparable with the situation in $\mathrm{Ni}_{1-y}\left(\mathrm{SiO}_{2}\right)_{y}$ descussed earlier.

The only exception in Figure 3, where such an exponential concentration dependence of $\sigma$, respectively $\sigma_{A}$, does not occur, is represented by the annealed $\mathrm{W}_{1-y}\left(\mathrm{Al}_{2} \mathrm{O}_{3}\right)_{y}$ samples. This phenomenon will be discussed in Sec. 3.3.

3) With increasing $y$ the temperature coefficient of resistivity, TCR, decreases and changes sign from positive to negative. [6] [11] [12] [14] [15] [34] The reason is an activation of localized electrons to the conduction band of the metallic phase. This conductivity contribution by activation is in competition with the positive contribution to the TCR due to scattering. The activation contribution is the larger the larger the amount of transferred electrons, i.e., the larger $y$, in correspondence to Equation (1).

In earlier papers it was suggested "that the GHE is a result of the drastic reduction of both the effective electron density and (in case of EHE) the effective carrier mobility”, (Pakhomov et al. [11]) or a drastic reduction of carrier density (Jing et al. [35]). These two suggestions [11] [35] correspond to our physical model summarized in Sec. I. We emphasise, however, that it is not any effective electron density or carrier density (electrons or holes), but it is the real electron density which is reduced in the M-I composites.

\subsection{The Effect of the Grain Size on the GHE}

Approaching the M-I transition, the charging energy arising from the positively charged metal ions grows more and more and one could assume that such 'metal' phase cannot exist, because the electrostatic contribution by the positive ions increases more and more as $n$ decreases. However, the growth of the electrostatic energy is not unbounded; decrease of $n$ is accompanied with a decrease of the sizes of the metal grains. For granular $\mathrm{Al}_{1-y} \mathrm{Ge}_{y}$ films, with increasing $y$ the sizes of the metal grains decrease from 10 - $20 \mathrm{~nm}$ (on the metallic-rich side) to sizes $<2 \mathrm{~nm}$ beyond the MIT (Rosenbaum et al. [36] [37]). This decrease of $D_{A}$ with decreasing metal content even continues in the dielectric regime, as found for $\mathrm{Ni}_{1-y}\left(\mathrm{SiO}_{2}\right)_{y}, \mathrm{Pt}_{1-y}\left(\mathrm{SiO}_{2}\right)_{y}$ and $\mathrm{Au}_{1-y}\left(\mathrm{Al}_{2} \mathrm{O}_{3}\right)_{y}$ thin films ([29], Fig. 17 therein), where $D_{A}$ decreases from $4 \mathrm{~nm}$ at $y \approx 0.5$ to $1 \mathrm{~nm}$ at $y \approx 0.9$. For co-sputtered granular $\mathrm{Ni}_{1-y}\left(\mathrm{SiO}_{2}\right)_{y}$ films, Abeles et al. found that the average particel size, $D_{A}$, decreases with Ni content: $D_{A}=14$ nm, $9.4 \mathrm{~nm}, 5.7 \mathrm{~nm}$, and $3.7 \mathrm{~nm}$ for 87, 67, 56 and $37 \mathrm{vol} \%$ Ni, respectively ([29], Fig. 11 therein).

We suppose that the electron transfer described by Equation (1), respectively Equation (20), holds also beyond the M-I transition. This assumption correlates with the concentration dependence of $D_{A}$, which decreases continuously through the M-I transition as cited.

As mentioned earlier ([18], Sec. IVA therein), Equation (1), is part and result of a complex energy balance realized during solidification of the alloy, where the sizes of the phase grains are part of this balance. Equation (1) holds for situations, where atomic diffusion does practically not play a role because of the high cooling rate during the film deposition process. Because of this suppression of the long-range diffusion, the EMT provides a more realistic description of the electrical properties of disordered alloys with phase separation than any percolation description. This is justified in [2] (Sec. IVA therein).

On the other hand, at sufficiently high temperatures, appreciable diffusion can take place leading to additional growth of $D_{A}$. With increasing $D_{A}$, for instance due to annealing, the electron transfer to the phase boundaries can no longer be expected to follow Equation (1). Otherwise, the growth of the electrostatic energy could be shoreless.

Therefore, the GHE decreases or disappears by annealing at sufficiently high temperatures [14]. This phenomenon is also reflected by the concentration dependences of $\sigma$ and $\sigma_{A}$ which can be essentially smaller than before annealing. One typical example is $\mathrm{W}_{1-y}\left(\mathrm{Al}_{2} \mathrm{O}_{3}\right)_{y}$, [29], Figure 3: Before annealing, $\log [\sigma(y) / \sigma(0)]$ is

${ }^{8}$ EHE is applied in [11] for the extraordinary Hall effect in magnetic M-I composites. 
approximately linear in $y /(1-y)$, but after annealing at $1200^{\circ} \mathrm{C}$ it is not. Reason is the fact that after annealing the metallic phase grains are essentially larger than before, for instance $D_{A} \approx 20 \mathrm{~nm}$ for $y \approx 0.47$ (Abeles et al. [38], Fig. 2 therein), whereas $D_{A} \approx 2 \mathrm{~nm}$ for the unannealed samples (Abeles et al. [38], Fig. 1 therein). Because of the large phase grains in the annealed $\mathrm{W}_{1-\mathrm{y}}\left(\mathrm{Al}_{2} \mathrm{O}_{3}\right)_{y}$ samples [38], the electron transfer (related to $n(0)$ ) is essentially smaller than in the unannealed samples. Elsewise, the electrostatic energy would be too large.

This can also explain the experimental finding [14] that the maximum of the enhancement of $R$ in $\mathrm{Zn}_{1-y}\left(\mathrm{SiO}_{2}\right)_{y}$ is about 60, but 700 in $\mathrm{Cu}_{1-y}\left(\mathrm{SiO}_{2}\right)_{y}$ : the size of the granules in $\mathrm{Zn}_{1-y}\left(\mathrm{SiO}_{2}\right)_{y}$ is much larger $\left(D_{A} \approx 20 \mathrm{~nm}\right.$, [14], p.608) than in $\mathrm{Cu}_{1-y}\left(\mathrm{SiO}_{2}\right)_{y}$, for which $D_{A} \approx 1 \mathrm{~nm}$ is given as the minimum value ([14], p. 606). Apparently, in $\mathrm{Zn}_{1-y}\left(\mathrm{SiO}_{2}\right)_{y}$ a certain measure of atomic diffusion has been happen during film deposition, so that this balance was shifted to smaller electron transfer, i.e., Equation (1) does no longer apply.

\section{Conclusions}

A formula is derived for the Hall coefficient $R$ of composites and applied to a discussion of the concentration dependence of $R$ in M-I composites. From the empirical relation $d \ln |R|=\alpha^{\prime} \cdot d \eta$ with $\eta=y /(1-y)$ found for experimental $R$ data of $\mathrm{Cu}_{1-y}\left(\mathrm{SiO}_{2}\right)_{y}$ and $\mathrm{Ni}_{1-y}\left(\mathrm{SiO}_{2}\right)_{y}$ thin films, it is concluded that both the GHE and the granular structure typical for M-I composites are caused by electron transfer from the metallic phase to the insulating phase which obeys $-d n=\beta \cdot n \cdot d\left(\frac{v_{B}}{v_{A}}\right)$. This equation holds for nanocomposites, where long-range atomic diffusion does practically not play a role during the film deposition process. It is part and result of a complex energy balance realized during solidification of the alloy, where the sizes of the phase grains are part of this balance.

In M-I composites, the decrease of electron density $n$ in the metallic phase occurs as interface charges occupying surface states on the insulating phase which is responsible for the granular structure.

\section{Acknowledgements}

The author is appreciative to MEAS Deutschland GmbH a TE Connectivity LTD company for supporting this work. He also would like to thank Professor Stolze from the University of Dortmund for a critical reading of the manuscript and Stefan Lange for technical support.

\section{References}

[1] Sonntag, J. (1989) Disordered Electronic Systems: Concentration Dependence of the dc Conductivity in Amorphous Transition-Metal-Metalloid Alloys (Metallic Regime). Physical Review B, 40, 3661-3671. http://dx.doi.org/10.1103/PhysRevB.40.3661

[2] Sonntag, J. (2005) Disordered Electronic Systems: II. Phase Separation and the Metal-Insulator Transition in Metal-Metalloid Alloys. Physical Review B, 71, 115114. http://dx.doi.org/10.1103/physrevb.71.115114

[3] Rowe, D.M. (2006) Thermoelectrics Handbook: Macro to Nano. CRC Press, Taylor and Francis Group, Boca Raton, London, New York.

[4] Minnich, A.J., Dresselhaus, M.S., Ren, Z.F. and Chen, G. (2009) Bulk Nanostructured Thermoelectric Materials: Current Research and Future Prospects. Energy \& Environmental Science, 2, 466-479. http://dx.doi.org/10.1039/b822664b

[5] Reddy, B. (2011) Nanotechnology and Nanomaterials. Advances in Diverse Industrial Applications of Nanocomposites. InTech.

[6] Zhao, B. and Yan, X. (1997) Giant Hall Effect in Granular Fe-SiO 2 Film. Journal of Applied Physics, 81, $4290-4292$. http://dx.doi.org/10.1063/1.364804

[7] Wu, Y.N., Li, Z.Q. and Lin, J.J. (2010) Giant Hall Effect in Nonmagnetic Mo/SnO ${ }_{2}$ Granular Films. Physical Review B, 82, Article ID: 092202. http://dx.doi.org/10.1103/PhysRevB.82.092202

[8] Wen, J.F., Wang, J.F., Zou, W.Q., Zhang, F.M. and Du, Y.W. (2005) Investigation on the Giant Hall Effect of $\left(\mathrm{Fe}_{x} \mathrm{Sn}_{100-x}\right)_{1-y}(\mathrm{SiO} 2)_{\mathrm{y}}$ Granular Films. Journal of Alloys and Compounds, 393, 77-80. http://dx.doi.org/10.1016/j.jallcom.2004.10.021

[9] Miao, G.X. and Xiao, G. (2004) Giant Hall Resistance in Pt-Based Ferromagnetic Alloys. Applied Physics Letters, 85, 73-75. http://dx.doi.org/10.1063/1.1757645 
[10] Pakhomov, A.B., Yan, X. and Zhao, B. (1995) Giant Hall Effect in Percolating Ferromagnetic Granular Metal-Insulator Films. Applied Physics Letters, 67, 3497-3499. http://dx.doi.org/10.1063/1.115259

[11] Pakhomov, A.B., Yan, X., Wang, N., Jing, X.N., Zhao, B., Fung, K.K., Xhie, J., Hung, T.F. and Wong, S.K. (1997) On the Origin of the Giant Hall Effect in Magnetic Granular Metals. Physica A, 241, 344-349. http://dx.doi.org/10.1016/S0378-4371(97)00105-2

[12] Zhang, X.X., Liu, H. and Pakhomov, A.B. (2000) Observation of Giant Hall Effect in Non-Magnetic Cermets. Physica B, 279, 81-83. http://dx.doi.org/10.1016/S0921-4526(99)00674-2

[13] Zhang, X.X., Wan, C., Liu, H., Li, Z.Q., Sheng, P. and Lin, J.J. (2001) Giant Hall Effect in Nonmagnetic Granular Metal Films. Physical Review Letters, 86, 5562-5565. http://dx.doi.org/10.1103/PhysRevLett.86.5562

[14] Liu, H., Zheng, R.K., Wen, G.H. and Zhang, X.X. (2004) Giant Hall Effect in Metal/Insulator Composite Films. Vacuum, 73, 603-610. http://dx.doi.org/10.1016/j.vacuum.2003.12.076

[15] Denardin, J.C., Knobel, M., Zhang, X.X. and Pakhomov, A.B. (2003) Giant Hall Effect in Superparamagnetic Granular Films. Journal of Magnetism and Magnetic Materials, 262, 15-22. http://dx.doi.org/10.1016/S0304-8853(03)00012-X

[16] Denardin, J.C., Pakhomov, A.B., Knobel, M., Liu, H. and Zhang, X.X. (2000) Giant Hall Effect in Co-SiO ${ }_{2} \mathrm{Nano}^{-}$ composites. Journal of Physics: Condensed Matter, 12, 3397-3399. http://dx.doi.org/10.1088/0953-8984/12/14/315

[17] Wan, C. and Sheng, P. (2002) Quantum Interference and the Giant Hall Effect in Percolating Systems. Physical Review B, 66, 075309. http://dx.doi.org/10.1103/PhysRevB.66.075309

[18] Sonntag, J. (2006) Disordered Electronic Systems: III. Thermoelectric Power in Alloys with Phase Separation. Physical Review B, 73, 045126. http://dx.doi.org/10.1103/physrevb.73.045126

[19] Sonntag, J. (2009) Thermoelectric Power in Alloys with Phase Separation (Composites). Journal of Physics: Condensed Matter, 21, 175703. http://dx.doi.org/10.1088/0953-8984/21/17/175703

[20] Savvides, N., Alister, S.P., Hurd, C.M. and Shiozaki, I. (1982) Localization in the Metallic Regime of Granular Cu$\mathrm{SiO}_{2}$ Films. Solid State Communications, 42, 143-145. http://dx.doi.org/10.1016/0038-1098(82)90370-2

[21] Cohen, M.H. and Jortner, J. (1973) Effective Medium Theory for the Hall Effect in Disordered Materials. Physical Review Letters, 30, 696-698. http://dx.doi.org/10.1103/PhysRevLett.30.696

[22] Czycholl, G. (2008) Theoretische Festkörperphysik. 3rd Edition, Springer-Verlag, Berlin.

[23] Kirejew, P.S. (1974) Physik der Halbleiter. Akademie-Verlag, Berlin.

[24] Kirejew, P.S. (1978) Semiconductor Physics. Mir Publishers, Moscow.

[25] Harman, T.C. and Honig, J.M. (1967) Thermoelectric and Thermomagnetic Effects and Applications. McGraw-Hill Book Company, New York.

[26] Cohen, M.H. and Jortner, J. (1974) The Inhomogeneous Transport Regime and Metal-Nonmetal Transitions in Disordered Material. Journal de Physique. Colloques, 35, 345-366. http://dx.doi.org/10.1051/jphyscol:1974467

[27] Odelevskii, V.I. (1951) Calculation of the Generalized Conductivity of Heterogeneous Systems. Journal of Technical Physics (USSR), 21, 678-685.

[28] Landauer, R. (1952) The Electrical Resistance of Binary Metallic Mixtures. Journal of Applied Physics, 23, $779-784$. http://dx.doi.org/10.1063/1.1702301

[29] Abeles, B., Sheng, P., Coutts, M.D. and Arie, Y. (1975) Structural and Electrical Properties of Granular Metal Films. Advances in Physics, 24, 407-461. http://dx.doi.org/10.1080/00018737500101431

[30] Schulze, G.E.R. (1967) Metallphysik. Akademie-Verlag, Berlin.

[31] Xiong, P., Xiao, G., Wang, J.Q., Xiao, J.Q., Jiang, J.S. and Chien, C.L. (1992) Extraordinary Hall Effect and Giant Magnetoresistance in Granular Co-Ag System. Physical Review Letters, 69, 3220-3224. http://dx.doi.org/10.1103/PhysRevLett.69.3220

[32] Tanaka, K., Saito, T., Suzuki, K. and Hasegawa, R. (1985) Role of Atomic Bonding for Compound and Glass Formation in Ni-Si, Pd-Si, and Ni-B Systems. Physical Review B, 32, 6853-6860. http://dx.doi.org/10.1103/PhysRevB.32.6853

[33] Priestley, E.B., Abeles, B. and Cohen, R.W. (1975) Surface Plasmons in Granular Ag-SiO 2 . Physical Review B, 12, 2121-2124. http://dx.doi.org/10.1103/PhysRevB.12.2121

[34] Ren, S.L., You, B., Du, J., Bai, X.J., Zhang, J., Zhang, W., Hu, A., Zhang, B. and Zhang, X.X. (2007) Magnetic Transport Properties in Iron/Iron-Oxide Films. Physica B: Condensed Matter, 400, 185-189. http://dx.doi.org/10.1016/j.physb.2007.07.005

[35] Jing, X.N., Wang, N., Pakhomov, A.B., Fung, K.K. and Yan, X. (1996) Effect of Annealing on the Giant Hall Effect. Physical Review B, 53, 14032-14035. http://dx.doi.org/10.1103/PhysRevB.53.14032 
[36] Rosenbaum, R.L., Slutzky, M., Möbius, A. and McLachlan, D.S. (1994) Various Methods for Determining the Critical Metallic Volume Fraction phi ${ }_{c}$ at the Metal-Insulator Transition. Journal of Physics: Condensed Matter, 6, 7977-7992. http://dx.doi.org/10.1088/0953-8984/6/39/018

[37] Lereah, Y., Deutscher, G. and Grünbaum, E. (1991) Formation of Dense Branching Morphology in the Crystallization of Al-Ge Amorphous Thin Films. Physical Review A, 44, 8316. http://dx.doi.org/10.1103/PhysRevA.44.8316

[38] Abeles, B., Pinch, H.L. and Gittleman, J.I. (1975) Percolation Conductivity in $\mathrm{W}-\mathrm{Al}_{2} \mathrm{O}_{3}$ Granular Metal Films. Physical Review Letters, 35, 247-250. http://dx.doi.org/10.1103/PhysRevLett.35.247

[39] Edwards, A.M., Fairbanks, M.C., Singh, A., Newport, R.J. and Gurman, S.J. (1989) An Investigation of the Structure of Amorphous $\mathrm{Si}_{1-x} \mathrm{Ni}_{x}$ through the Metal-Insulator Transition. Physica B: Condensed Matter, 158, 600-601. http://dx.doi.org/10.1016/0921-4526(89)90402-X

[40] Edwards, A.M., Fairbanks, M.C. and Newport, R.J. (1991) Structural Studies of Amorphous Ge-Au Alloys. Philosophical Magazine Part B, 63, 457-463. http://dx.doi.org/10.1080/13642819108205950

[41] Lorentz, R.D., Bienenstock, A. and Morrison, T.I. (1994) Structural Studies of the Phase Separation of Amorphous $\mathrm{Fe}_{x} \mathrm{Ge}_{1-x}$ Alloys. Physical Review B, 49, 3172-3182. http://dx.doi.org/10.1103/PhysRevB.49.3172

[42] Regan, M.J., Rice, M., van Raap, M.B.F. and Bienenstock, A. (1994) Anisotropic Phase Separation through the Metal-Insulator Transition in Amorphous Alloys. Physical Review Letters, 73, 1118-1121. http://dx.doi.org/10.1103/PhysRevLett.73.1118

[43] Van Raap, M.B.F., Regan, M.J. and Bienenstock, A. (1995) Evidence of Phase Separation in Amorphous Fe $\mathrm{Si}_{1-x} \mathrm{Films}$ Journal of Non-Crystalline Solids, 191, 155-163. http://dx.doi.org/10.1016/0022-3093(95)00286-3

[44] Yoshizumi, S., Mael, D., Geballe, T.H. and Greene, R.L. (1985) The Metal-Insulator Transition and Superconductivity in Amorphous Molybdenum-Germanium Alloys. In: Fritzsche, H. and Adler, D., Eds., Localization and MetalInsulator Transitions, Plenum Press, Berlin, 77-88. http://dx.doi.org/10.1007/978-1-4613-2517-8_7

[45] Mael, D., Yoshizumi, S. and Geballe, T.H. (1986) Specific Heat of Amorphous $\mathrm{Mo}_{x} \mathrm{Ge}_{1-x}$ through the Metal-Insulator Transition. Physical Review B, 34, 467-470. http://dx.doi.org/10.1103/PhysRevB.34.467

[46] Fischer, J. and Löneysen, H.V. (1993) Electronic Properties and Superconductivity of Amorphous $\mathrm{Si}_{1-x} \mathrm{Au}_{x}$ Alloys near the Metal-Insulator Transition. Annalen der Physik, 2, 635-646. http://dx.doi.org/10.1002/andp.19935050705

[47] Mizutani, U., Ishizuka, T. and Fukunaga, T. (1997) Interrelations of Atomic Structures, Electronic Structures, Electron Transport, and Magnetic Properties across the Metal-Insulator Transition for Amorphous $\mathrm{V}_{x} \mathrm{Si}_{100-x}(7 \leq x \leq 74)$ Alloys. Journal of Physics: Condensed Matter, 9, 5333-5354. http://dx.doi.org/10.1088/0953-8984/9/25/004

[48] Rogatchev, A.Y., Takeuchi, T. and Mizutani, U. (2000) Comparison of the Specific Heat and the Conductivity of Amorphous $\mathrm{Ti}_{x} \mathrm{Si}_{100-x}$ Alloys across the Metal-Insulator Transition. Physical Review B, 61, 10010-10014. http://dx.doi.org/10.1103/PhysRevB.61.10010

[49] Abkemeier, K.M., Adkins, C.J., Asal, R. and Davis, E.A. (1992) Conductivity and Magnetoresistance of Hydrogenated Amorphous Silicon-Nickel Alloys near the Metal-Insulator Transition. Journal of Physics: Condensed Matter, 4, 91139130. http://dx.doi.org/10.1088/0953-8984/4/46/017

[50] Abkemeier, K.M., Adkins, C.J., Asal, R. and Davis, E.A. (1992) Hopping Conduction in Hydrogenated Amorphous $\mathrm{Si}_{1-y} \mathrm{Ni}_{y}$. Philosophical Magazine Part B, 65, 675-680. http://dx.doi.org/10.1080/13642819208204902

\section{Submit or recommend next manuscript to SCIRP and we will provide best service for you:}

Accepting pre-submission inquiries through Email, Facebook, Linkedin, Twitter, etc

A wide selection of journals (inclusive of 9 subjects, more than 200 journals)

Providing a 24-hour high-quality service

User-friendly online submission system

Fair and swift peer-review system

Efficient typesetting and proofreading procedure

Display of the result of downloads and visits, as well as the number of cited articles

Maximum dissemination of your research work

Submit your manuscript at: http://papersubmission.scirp.org/ 